\title{
Existence of nonoscillatory solutions to nonlinear higher-order neutral dynamic equations
}

\author{
Yang-Cong Qiu ${ }^{1}$, Kuo-Shou Chiu ${ }^{2 *}$ (D), Irena Jadlovská3 and Tongxing Li
}

"Correspondence: kschiu@umce.cl 2Departamento de Matemática, Facultad de Ciencias Básicas, Universidad Metropolitana de Ciencias de la Educación, Santiago, Chile

Full list of author information is available at the end of the article

\begin{abstract}
We investigate the existence of different types of nonoscillatory solutions to a class of higher-order nonlinear neutral dynamic equations on a time scale. Two examples are provided to show the significance of the conclusions.
\end{abstract}

MSC: 34K11;34N05; 39A13

Keywords: Nonoscillatory solution; Nonlinear neutral dynamic equation; Higher-order; Time scale

\section{Introduction}

The time scale theory has been introduced and developed rapidly since 1988; see, for instance, $[1-4,7,8]$. Afterwards, many scholars were concerned with the oscillation of dynamic equations on time scales and they obtained abundant achievements. Besides, some research on the existence and asymptotic behavior of nonoscillatory solutions to dynamic equations on time scales has been also improved recently, we refer the reader to [5, 6, 915].

Since 2007, numerous researchers have investigated the existence of nonoscillatory solutions to several classes of nonlinear neutral dynamic equations

$$
\begin{aligned}
& {[x(t)+p(t) x(g(t))]^{\Delta}+f(t, x(h(t)))=0,} \\
& {\left[r(t)(x(t)+p(t) x(g(t)))^{\Delta}\right]^{\Delta}+f(t, x(h(t)))=0,}
\end{aligned}
$$

and

$$
\left(r_{1}(t)\left(r_{2}(t)(x(t)+p(t) x(g(t)))^{\Delta}\right)^{\Delta}\right)^{\Delta}+f(t, x(h(t)))=0,
$$

successively. Zhu and Wang [15] studied (1) and presented the existence of nonoscillatory solutions by using a Banach space and Krasnoselskii's fixed point theorem. Actually, the authors gave a general way to establish the existence of nonoscillatory solutions to (1). Inspired by [15], Gao and Wang [6] were concerned with (2) under the condition

(c) The Author(s) 2020. This article is licensed under a Creative Commons Attribution 4.0 International License, which permits use, sharing, adaptation, distribution and reproduction in any medium or format, as long as you give appropriate credit to the original author(s) and the source, provide a link to the Creative Commons licence, and indicate if changes were made. The images or other third party material in this article are included in the article's Creative Commons licence, unless indicated otherwise in a credit line to the material. If material is not included in the article's Creative Commons licence and your intended use is not permitted by statutory regulation or exceeds the permitted use, you will need to obtain permission directly from the copyright holder. To view a copy of this licence, visit http://creativecommons.org/licenses/by/4.0/. 
$\int_{t_{0}}^{\infty} 1 / r(t) \Delta t<\infty$. Similar to the results in [15], there are two types of asymptotic behavior of eventually positive solutions to (2). Later on, Deng and Wang [5] considered (2) with another condition $\int_{t_{0}}^{\infty} 1 / r(t) \Delta t=\infty$, and summarized four types of eventually positive solutions to (2). It is clear to see that the asymptotic behavior of eventually positive solutions in [5] are more complex than that of [6]. From [5, 6], we can see the fact that the existence and asymptotic behavior of nonoscillatory solutions are greatly different for various kinds of the integral convergence and divergence of the reciprocals of the coefficients $r_{i}$ in equations. To find a general relationship between these factors, some researches have been performed.

For the third-order nonlinear neutral dynamic equation (3), there exist four cases for the convergence and divergence of the integrals $\int_{t_{0}}^{\infty} 1 / r_{1}(t) \Delta t$ and $\int_{t_{0}}^{\infty} 1 / r_{2}(t) \Delta t$ :

(B1) $\int_{t_{0}}^{\infty} 1 / r_{1}(t) \Delta t=\int_{t_{0}}^{\infty} 1 / r_{2}(t) \Delta t=\infty$, see Qiu [10];

(B2) $\int_{t_{0}}^{\infty} 1 / r_{1}(t) \Delta t<\infty$ and $\int_{t_{0}}^{\infty} 1 / r_{2}(t) \Delta t<\infty$, see Qiu and Wang [13];

(B3) $\int_{t_{0}}^{\infty} 1 / r_{1}(t) \Delta t=\infty$ and $\int_{t_{0}}^{\infty} 1 / r_{2}(t) \Delta t<\infty$, see Qiu et al. [14];

(B4) $\int_{t_{0}}^{\infty} 1 / r_{1}(t) \Delta t<\infty$ and $\int_{t_{0}}^{\infty} 1 / r_{2}(t) \Delta t=\infty$, see Qiu et al. [12].

In particular, Qiu and Wang [13] considered a higher-order nonlinear neutral dynamic equation

$$
R_{n}(t, x(t))+f(t, x(h(t)))=0
$$

under the condition $\int_{t_{0}}^{\infty} 1 / r_{i}(t) \Delta t<\infty, i=1,2, \ldots, n-1$, where $n \geq 3$ and

$$
R_{k}(t, x(t))= \begin{cases}x(t)+p(t) x(g(t)), & k=0, \\ r_{n-k}(t) R_{k-1}^{\Delta}(t, x(t)), & 1 \leq k \leq n-1, \\ R_{n-1}^{\Delta}(t, x(t)), & k=n .\end{cases}
$$

Moreover, Qiu et al. [11] studied (4) with

$$
\int_{t_{0}}^{\infty} \frac{\Delta t}{r_{1}(t)}=\infty \quad \text { and } \quad \int_{t_{0}}^{\infty} \frac{\Delta t}{r_{i}(t)}=M_{i}<\infty, \quad i=2,3, \ldots, n-1 .
$$

In this paper, we continue the study on the existence of nonoscillatory solutions to (4) on a time scale $\mathbb{T}$, where $n \geq 3$, sup $\mathbb{T}=\infty$, and $t \in\left[t_{0}, \infty\right)_{\mathbb{T}}$ with $t_{0} \in \mathbb{T}$. In addition, the following assumptions are supposed to hold:

(C1) $r_{i} \in \mathrm{C}_{\mathrm{rd}}\left(\left[t_{0}, \infty\right)_{\mathbb{T}},(0, \infty)\right), i=1,2, \ldots, n-1$, and there exist constants $M_{i}>0$, $i=1,2, \ldots, n-2$ such that

$$
\int_{t_{0}}^{\infty} \frac{\Delta t}{r_{i}(t)}=M_{i}<\infty, \quad i=1,2, \ldots, n-2, \quad \text { and } \quad \int_{t_{0}}^{\infty} \frac{\Delta t}{r_{n-1}(t)}=\infty
$$

(C2) $p \in \mathrm{C}_{\mathrm{rd}}\left(\left[t_{0}, \infty\right)_{\mathbb{T}}, \mathbb{R}\right)$ and $\lim _{t \rightarrow \infty} p(t)=p_{0}$, where $\left|p_{0}\right|<1$;

(C3) $g, h \in \mathrm{C}_{\mathrm{rd}}\left(\left[t_{0}, \infty\right)_{\mathbb{T}}, \mathbb{T}\right), g(t) \leq t, \lim _{t \rightarrow \infty} g(t)=\lim _{t \rightarrow \infty} h(t)=\infty$, and $\lim _{t \rightarrow \infty} R(g(t)) / R(t)=\eta \in(0,1]$, where $R(t)=1+\int_{t_{0}}^{t} 1 / r_{n-1}(s) \Delta s$; if $p_{0} \in(-1,0]$, there exists a sequence $\left\{c_{k}\right\}_{k \geq 0}$ such that $\lim _{k \rightarrow \infty} c_{k}=\infty$ and $g\left(c_{k+1}\right)=c_{k}$;

(C4) $f \in \mathrm{C}\left(\left[t_{0}, \infty\right)_{\mathbb{T}} \times \mathbb{R}, \mathbb{R}\right), f(t, x)$ is nondecreasing in $x$, and $x f(t, x)>0$ for $x \neq 0$.

By employing an appropriate Banach space and Krasnoselskii's fixed point theorem, we present the existence of different kinds of nonoscillatory solutions to (4). Finally, two examples are shown to illustrate our conclusions. 


\section{Auxiliary results}

We denote all continuous functions mapping $\left[T_{0}, \infty\right)_{\mathbb{T}}$ into $\mathbb{R}$ by $C\left(\left[T_{0}, \infty\right)_{\mathbb{T}}, \mathbb{R}\right)$. Then define a Banach space

$$
\mathrm{BC}_{\lambda}\left[T_{0}, \infty\right)_{\mathbb{T}}=\left\{x \in \mathrm{C}\left(\left[T_{0}, \infty\right)_{\mathbb{T}}, \mathbb{R}\right): \sup _{t \in\left[T_{0}, \infty\right)_{\mathbb{T}}}\left|\frac{x(t)}{R^{2 \lambda}(t)}\right|<\infty\right\}
$$

with $\|x\|_{\lambda}=\sup _{t \in\left[T_{0}, \infty\right)_{\mathbb{T}}}\left|x(t) / R^{2 \lambda}(t)\right|$ for $\lambda=0$, 1. In the sequel, define $z(t)=x(t)+p(t) x(g(t))$ for simplicity, and we just consider the eventually positive solutions to (4). Now, a lemma is presented to show the relationship between the functions $z$ and $x$. The proof is similar to the one in [5, Lemma 2.3] and so is omitted.

Lemma 2.1 Suppose that $x$ is an eventually positive solution to $(4)$ and $\lim _{t \rightarrow \infty} z(t) / R^{\lambda}(t)=$ a for $\lambda=0,1$. Then we have

$$
\lim _{t \rightarrow \infty} \frac{x(t)}{R^{\lambda}(t)}=\frac{a}{1+p_{0} \eta^{\lambda}}
$$

if a is finite, and

$$
\limsup _{t \rightarrow \infty} \frac{x(t)}{R^{\lambda}(t)}=\infty
$$

if a is infinite.

Next, we divide all eventually positive solutions to (4) into four groups.

Theorem 2.2 If $x$ is an eventually positive solution to (4), then one of the following four cases holds:

$$
\begin{aligned}
& \text { (A1) } x \in A(0,0) \text {; } \\
& \text { (A2) } x \in A(b, 0) \text {; } \\
& \text { (A3) } x \in A(\infty, b) \text {; } \\
& \text { (A4) } \limsup _{t \rightarrow \infty} x(t)=\infty \text { and } \lim _{t \rightarrow \infty} x(t) / R(t)=0 .
\end{aligned}
$$

Here,

$$
A(\alpha, \beta)=\left\{x \in S: \lim _{t \rightarrow \infty} x(t)=\alpha, \lim _{t \rightarrow \infty} \frac{x(t)}{R(t)}=\beta\right\},
$$

$S$ is the set of all eventually positive solutions of (4), and $b$ is a positive constant.

Proof Assume that $x$ is an eventually positive solution to (4). From (C2) and (C3), there exist a $t_{1} \in\left[t_{0}, \infty\right)_{\mathbb{T}}$ and a $p_{1}$ satisfying $\left|p_{0}\right|<p_{1}<1$ such that $x(t)>0, x(g(t))>0, x(h(t))>0$, and $|p(t)| \leq p_{1}$ for $t \in\left[t_{1}, \infty\right)_{\mathbb{T}}$. For $t \in\left[t_{1}, \infty\right)_{\mathbb{T}}$, according to (4) and (C4), we have

$$
R_{n-1}^{\Delta}(t, x(t))=-f(t, x(h(t)))<0,
$$

which means that $R_{n-1}$ is strictly decreasing on $\left[t_{1}, \infty\right)_{\mathbb{T}}$. Moreover, it follows that

$$
R_{n-2}^{\Delta}(t, x(t)) \leq \frac{r_{1}\left(t_{1}\right) R_{n-2}^{\Delta}\left(t_{1}, x\left(t_{1}\right)\right)}{r_{1}(t)}, \quad t \in\left[t_{1}, \infty\right)_{\mathbb{T}} .
$$


If there exists a $T \in\left[t_{1}, \infty\right)_{\mathbb{T}}$ such that $R_{n-2}^{\Delta}(T, x(T)) \leq 0$, then by (6) we know that $R_{n-2}^{\Delta}$ is eventually negative. Otherwise, we arrive at $R_{n-2}^{\Delta}(t, x(t))>0$ for all $t \in\left[t_{1}, \infty\right)_{\mathbb{T}}$. Hence, $R_{n-2}$ is always eventually monotonic. Letting $t$ be replaced by $s$ and integrating (6) from $t_{1}$ to $t, t \in\left[\sigma\left(t_{1}\right), \infty\right)_{\mathbb{T}}$, by $(\mathrm{C} 1)$ we obtain

$$
\begin{aligned}
R_{n-2}(t, x(t))-R_{n-2}\left(t_{1}, x\left(t_{1}\right)\right) & \leq r_{1}\left(t_{1}\right) R_{n-2}^{\Delta}\left(t_{1}, x\left(t_{1}\right)\right) \int_{t_{1}}^{t} \frac{\Delta s}{r_{1}(s)} \\
& <r_{1}\left(t_{1}\right)\left|R_{n-2}^{\Delta}\left(t_{1}, x\left(t_{1}\right)\right)\right| \cdot M_{1}
\end{aligned}
$$

which means that $R_{n-2}$ is upper bounded. When $n=3$, we see that $r_{2} z^{\Delta}$ is eventually monotonic and upper bounded. When $n \geq 4$, since $r_{2} R_{n-3}^{\Delta}$ is eventually monotonic, it follows that $r_{2} R_{n-3}^{\Delta}$ and $R_{n-3}^{\Delta}$ are eventually positive or eventually negative. Thus, $R_{n-3}$ is eventually monotonic.

Since $R_{n-2}$ is upper bounded, there exist a constant $c_{1}$ and a $t_{2} \in\left[t_{1}, \infty\right)_{\mathbb{T}}$ such that $R_{n-2}(t, x(t)) \leq c_{1}$ for $t \in\left[t_{2}, \infty\right)_{\mathbb{T}}$, that is,

$$
R_{n-3}^{\Delta}(t, x(t)) \leq \frac{c_{1}}{r_{2}(t)}
$$

Substituting $s$ for $t$ and integrating (7) from $t_{2}$ to $t, t \in\left[\sigma\left(t_{2}\right), \infty\right)_{\mathbb{T}}$, we have

$$
\begin{aligned}
R_{n-3}(t, x(t)) & \leq R_{n-3}\left(t_{2}, x\left(t_{2}\right)\right)+c_{1} \int_{t_{2}}^{t} \frac{\Delta s}{r_{2}(s)} \\
& <R_{n-3}\left(t_{2}, x\left(t_{2}\right)\right)+\left|c_{1}\right| \cdot M_{2},
\end{aligned}
$$

which implies that $R_{n-3}$ is upper bounded. When $n=4$, we see that $r_{3} z^{\Delta}$ is eventually monotonic and upper bounded. By analogy, for all $n \geq 3$, it always satisfies the requirement that $r_{n-1} z^{\Delta}$ is eventually monotonic and upper bounded. Then we need to consider two cases.

Case 1. $r_{n-1} z^{\Delta}$ is eventually strictly decreasing. We can claim that

$$
0 \leq \lim _{t \rightarrow \infty} r_{n-1}(t) z^{\Delta}(t)=L_{1}<\infty
$$

Otherwise, there exist a constant $c_{2}<0$ and a $t_{3} \in\left[t_{2}, \infty\right)_{\mathbb{T}}$ such that

$$
z^{\Delta}(t) \leq \frac{c_{2}}{r_{n-1}(t)}, \quad t \in\left[t_{3}, \infty\right)_{\mathbb{T}}
$$

Letting $t$ be replaced by $s$ and integrating (9) from $t_{3}$ to $t, t \in\left[\sigma\left(t_{3}\right), \infty\right)_{\mathbb{T}}$, by $(\mathrm{C} 1)$ we have

$$
z(t) \leq z\left(t_{3}\right)+c_{2} \int_{t_{3}}^{t} \frac{\Delta s}{r_{n-1}(s)} \rightarrow-\infty
$$

as $t \rightarrow \infty$. Then we get $p_{0} \in(-1,0]$, and thus there exists a $t_{4} \in\left[t_{3}, \infty\right)_{\mathbb{T}}$ such that $x(t)<$ $-p(t) x(g(t))<p_{1} x(g(t))$ for $t \in\left[t_{4}, \infty\right)_{\mathbb{T}}$. In view of (C3), there exists a positive integer $N$ satisfying $c_{k} \in\left[t_{4}, \infty\right)_{\mathbb{T}}$ for all $k \geq N$. Moreover, for any $k \geq N+1$,

$$
x\left(c_{k}\right)<p_{1} x\left(c_{k-1}\right)<p_{1}^{2} x\left(c_{k-2}\right)<\cdots<p_{1}^{k-N} x\left(c_{N}\right)
$$


which means that $\lim _{k \rightarrow \infty} x\left(c_{k}\right)=0$ and thus $\lim _{k \rightarrow \infty} z\left(c_{k}\right)=0$. It is in contradiction with $\lim _{t \rightarrow \infty} z(t)=-\infty$. Therefore, (8) holds.

If $L_{1}>0$, then we have $z^{\Delta}(s)>L_{1} / r_{n-1}(s)$ for $s \in\left[t_{2}, \infty\right)_{\mathbb{T}}$. Integrating this inequality from $t_{2}$ to $t, t \in\left[\sigma\left(t_{2}\right), \infty\right)_{\mathbb{T}}$, we obtain

$$
z(t)>z\left(t_{2}\right)+L_{1} \int_{t_{2}}^{t} \frac{\Delta s}{r_{n-1}(s)} \rightarrow \infty
$$

as $t \rightarrow \infty$.

If $L_{1}=0$, since $r_{n-1} z^{\Delta}$ and $z^{\Delta}$ are both eventually positive, then it follows that $z$ is eventually strictly increasing. From the above, we know that $\lim _{t \rightarrow \infty} z(t)<0$ does not hold. Therefore, we get

$$
0 \leq \lim _{t \rightarrow \infty} z(t)=L_{0} \leq \infty
$$

Case 2. $r_{n-1} z^{\Delta}$ is eventually strictly increasing, which means that $r_{n-1} z^{\Delta}$ is eventually positive or eventually negative.

If $r_{n-1} z^{\Delta}$ is eventually positive, since it is also upper bounded, then there exists a constant $c_{3}>0$ such that $\lim _{t \rightarrow \infty} r_{n-1}(t) z^{\Delta}(t)=c_{3}$. Hence, there exists a $t_{3} \in\left[t_{2}, \infty\right)_{\mathbb{T}}$ satisfying that

$$
z^{\Delta}(t) \geq \frac{c_{3}}{2 r_{n-1}(t)}, \quad t \in\left[t_{3}, \infty\right)_{\mathbb{T}}
$$

Substituting $s$ for $t$ and integrating (10) from $t_{3}$ to $t, t \in\left[\sigma\left(t_{3}\right), \infty\right)_{\mathbb{T}}$, we have

$$
z(t) \geq z\left(t_{3}\right)+\frac{c_{3}}{2} \int_{t_{3}}^{t} \frac{\Delta s}{r_{n-1}(s)} \rightarrow \infty
$$

as $t \rightarrow \infty$.

If $r_{n-1} z^{\Delta}$ is eventually negative, then it follows that $\lim _{t \rightarrow \infty} r_{n-1}(t) z^{\Delta}(t) \leq 0$. From the above, it means that $\lim _{t \rightarrow \infty} r_{n-1}(t) z^{\Delta}(t)=0$ and so $z^{\Delta}(t)<0$ for $t \in\left[t_{2}, \infty\right)_{\mathbb{T}}$. Moreover, we get

$$
0 \leq \lim _{t \rightarrow \infty} z(t)=L_{0}<\infty
$$

Employing the L'Hôpital's rule in [3, Theorem 1.120], we obtain

$$
\lim _{t \rightarrow \infty} r_{n-1}(t) z^{\Delta}(t)=\lim _{t \rightarrow \infty} \frac{z(t)}{R(t)}=L_{1}
$$

By virtue of Lemma 2.1, it is clear that one of the cases (A1)-(A4) holds. This completes the proof.

\section{Main results}

In this section, the existence of eventually positive solutions to (4) is presented. Now, we show a sufficient and necessary condition for the type $A(\infty, b)$. 
Theorem 3.1 Equation (4) has an eventually positive solution $x \in A(\infty, b)$ if and only if there exists a constant $K>0$ such that

$$
\int_{t_{0}}^{\infty} \int_{t_{0}}^{u_{n-2}} \int_{t_{0}}^{u_{n-3}} \cdots \int_{t_{0}}^{u_{1}} \frac{f\left(u_{0}, K R\left(h\left(u_{0}\right)\right)\right)}{\prod_{i=1}^{n-2} r_{i}\left(u_{i}\right)} \Delta u_{0} \Delta u_{1} \cdots \Delta u_{n-2}<\infty
$$

where $b$ is a positive constant.

Proof Suppose that $x$ is an eventually positive solution to (4) satisfying $x \in A(\infty, b)$. By Lemma 2.1 we claim that

$$
\lim _{t \rightarrow \infty} z(t)=\infty \text { and } \quad \lim _{t \rightarrow \infty} r_{n-1}(t) z^{\Delta}(t)=\lim _{t \rightarrow \infty} \frac{z(t)}{R(t)}=\left(1+p_{0} \eta\right) b
$$

If $\lim _{t \rightarrow \infty} z(t)<\infty$, then it will cause $\lim _{t \rightarrow \infty} x(t)<\infty$, which contradicts $x \in A(\infty, b)$. Hence, there exists a $t_{1} \in\left[t_{0}, \infty\right)_{\mathbb{T}}$ such that $x(t)>0, x(g(t))>0$, and $x(h(t)) \geq b R(h(t)) / 2$ for $t \in\left[t_{1}, \infty\right)_{\mathbb{T}}$. Substituting $u_{0}$ for $t$ into (4) and integrating it from $t_{1}$ to $u_{1}, u_{1} \in\left[\sigma\left(t_{1}\right), \infty\right)_{\mathbb{T}}$, we have

$$
R_{n-1}\left(u_{1}, x\left(u_{1}\right)\right)-R_{n-1}\left(t_{1}, x\left(t_{1}\right)\right)=-\int_{t_{1}}^{u_{1}} f\left(u_{0}, x\left(h\left(u_{0}\right)\right)\right) \Delta u_{0}
$$

that is,

$$
R_{n-2}^{\Delta}\left(u_{1}, x\left(u_{1}\right)\right)=\frac{R_{n-1}\left(t_{1}, x\left(t_{1}\right)\right)}{r_{1}\left(u_{1}\right)}-\frac{1}{r_{1}\left(u_{1}\right)} \int_{t_{1}}^{u_{1}} f\left(u_{0}, x\left(h\left(u_{0}\right)\right)\right) \Delta u_{0} .
$$

Integrating (12) from $t_{1}$ to $u_{2}, u_{2} \in\left[\sigma\left(t_{1}\right), \infty\right)_{\mathbb{T}}$, we obtain

$$
\begin{aligned}
R_{n-2}\left(u_{2}, x\left(u_{2}\right)\right)-R_{n-2}\left(t_{1}, x\left(t_{1}\right)\right)= & R_{n-1}\left(t_{1}, x\left(t_{1}\right)\right) \int_{t_{1}}^{u_{2}} \frac{\Delta u_{1}}{r_{1}\left(u_{1}\right)} \\
& -\int_{t_{1}}^{u_{2}} \int_{t_{1}}^{u_{1}} \frac{f\left(u_{0}, x\left(h\left(u_{0}\right)\right)\right)}{r_{1}\left(u_{1}\right)} \Delta u_{0} \Delta u_{1} .
\end{aligned}
$$

Analogously, for all $n \geq 3$, it follows that

$$
\begin{aligned}
& r_{n-1}\left(u_{n-1}\right) z^{\Delta}\left(u_{n-1}\right)-r_{n-1}\left(t_{1}\right) z^{\Delta}\left(t_{1}\right) \\
& =\sum_{k=2}^{n-1} R_{k}\left(t_{1}, x\left(t_{1}\right)\right) \int_{t_{1}}^{u_{n-1}} \int_{t_{1}}^{u_{n-2}} \cdots \int_{t_{1}}^{u_{n-k+1}} \frac{\Delta u_{n-k} \Delta u_{n-k+1} \cdots \Delta u_{n-2}}{\prod_{i=n-k}^{n-2} r_{i}\left(u_{i}\right)} \\
& \quad-\int_{t_{1}}^{u_{n-1}} \int_{t_{1}}^{u_{n-2}} \cdots \int_{t_{1}}^{u_{1}} \frac{f\left(u_{0}, x\left(h\left(u_{0}\right)\right)\right)}{\prod_{i=1}^{n-2} r_{i}\left(u_{i}\right)} \Delta u_{0} \Delta u_{1} \cdots \Delta u_{n-2} .
\end{aligned}
$$

Letting $u_{n-1} \rightarrow \infty$, for all $2 \leq k \leq n-1$, by (C1) we have

$$
\begin{aligned}
& \int_{t_{1}}^{\infty} \int_{t_{1}}^{u_{n-2}} \int_{t_{1}}^{u_{n-3}} \cdots \int_{t_{1}}^{u_{n-k+1}} \frac{\Delta u_{n-k} \Delta u_{n-k+1} \cdots \Delta u_{n-2}}{\prod_{i=n-k}^{n-2} r_{i}\left(u_{i}\right)} \\
& \quad<\int_{t_{1}}^{\infty} \int_{t_{1}}^{\infty} \int_{t_{1}}^{\infty} \cdots \int_{t_{1}}^{\infty} \frac{\Delta u_{n-k} \Delta u_{n-k+1} \cdots \Delta u_{n-2}}{\prod_{i=n-k}^{n-2} r_{i}\left(u_{i}\right)} \leq \prod_{i=n-k}^{n-2} M_{i}<\infty .
\end{aligned}
$$


Hence, by virtue of (C4) and (13), it is not difficult to see that

$$
\begin{aligned}
& \int_{t_{1}}^{\infty} \int_{t_{1}}^{u_{n-2}} \int_{t_{1}}^{u_{n-3}} \cdots \int_{t_{1}}^{u_{1}} \frac{f\left(u_{0}, b R\left(h\left(u_{0}\right)\right) / 2\right)}{\prod_{i=1}^{n-2} r_{i}\left(u_{i}\right)} \Delta u_{0} \Delta u_{1} \cdots \Delta u_{n-2} \\
& \quad \leq \int_{t_{1}}^{\infty} \int_{t_{1}}^{u_{n-2}} \int_{t_{1}}^{u_{n-3}} \cdots \int_{t_{1}}^{u_{1}} \frac{f\left(u_{0}, x\left(h\left(u_{0}\right)\right)\right)}{\prod_{i=1}^{n-2} r_{i}\left(u_{i}\right)} \Delta u_{0} \Delta u_{1} \cdots \Delta u_{n-2}<\infty,
\end{aligned}
$$

which implies that (11) holds.

On the contrary, if there exists a constant $K>0$ such that (11) holds, then there are two cases to be considered.

Case $1.0 \leq p_{0}<1$. When $p_{0}>0$, choose a constant $p_{1}$ satisfying $p_{0}<p_{1}<\left(1+4 p_{0}\right) / 5<1$. In view of $(\mathrm{C} 2)$ and (11), there exists a $T_{0} \in\left[t_{0}, \infty\right)_{\mathbb{T}}$ such that for $t \in\left[T_{0}, \infty\right)_{\mathbb{T}}$,

$$
p(t)>0, \quad \frac{5 p_{1}-1}{4} \leq p(t) \leq p_{1}<1, \quad p(t) \frac{R(g(t))}{R(t)} \geq \frac{5 p_{1}-1}{4} \eta
$$

and

$$
\int_{T_{0}}^{\infty} \int_{T_{0}}^{u_{n-2}} \int_{T_{0}}^{u_{n-3}} \cdots \int_{T_{0}}^{u_{1}} \frac{f\left(u_{0}, K R\left(h\left(u_{0}\right)\right)\right)}{\prod_{i=1}^{n-2} r_{i}\left(u_{i}\right)} \Delta u_{0} \Delta u_{1} \cdots \Delta u_{n-2} \leq \frac{\left(1-p_{1} \eta\right) K}{8} .
$$

When $p_{0}=0$, choose $p_{1}$ satisfying $|p(t)| \leq p_{1} \leq 1 / 13$ for $t \in\left[T_{0}, \infty\right)_{\mathbb{T}}$. By $(\mathrm{C} 3)$, there always exists a $T_{1} \in\left(T_{0}, \infty\right)_{\mathbb{T}}$ such that $g(t) \geq T_{0}$ and $h(t) \geq T_{0}$ for $t \in\left[T_{1}, \infty\right)_{\mathbb{T}}$.

Define a Banach space $\mathrm{BC}_{1}\left[T_{0}, \infty\right)_{\mathbb{T}}$ as $(5)$,

$$
\Omega_{1}=\left\{x \in \mathrm{BC}_{1}\left[T_{0}, \infty\right)_{\mathbb{T}}: \frac{K}{2} R(t) \leq x(t) \leq K R(t)\right\}
$$

and two operators $U_{1}$ and $V_{1}: \Omega_{1} \rightarrow \mathrm{BC}_{1}\left[T_{0}, \infty\right)_{\mathbb{T}}$ as follows:

$$
\begin{aligned}
& \left(U_{1} x\right)(t)= \begin{cases}3 K p_{1} \eta R(t) / 4-p\left(T_{1}\right) x\left(g\left(T_{1}\right)\right) R(t) / R\left(T_{1}\right), & t \in\left[T_{0}, T_{1}\right)_{\mathbb{T}}, \\
3 K p_{1} \eta R(t) / 4-p(t) x(g(t)), & t \in\left[T_{1}, \infty\right)_{\mathbb{T}},\end{cases} \\
& \left(V_{1} x\right)(t)= \begin{cases}3 K R(t) / 4, & t \in\left[T_{0}, T_{1}\right)_{\mathbb{T}}, \\
3 K R(t) / 4+\int_{T_{1}}^{t} \int_{u_{n-1}}^{\infty} \int_{T_{1}}^{u_{n-2}} \int_{T_{1}}^{u_{n-3}} \cdots \int_{T_{1}}^{u_{1}} \frac{f\left(u_{0}, x\left(h\left(u_{0}\right)\right)\right)}{\prod_{i=1}^{n-1} r_{i}\left(u_{i}\right)} \Delta u_{0} \Delta u_{1} \cdots \Delta u_{n-1}, \\
t \in\left[T_{1}, \infty\right)_{\mathbb{T}} .\end{cases}
\end{aligned}
$$

The proof that $U_{1}$ and $V_{1}$ satisfy the conditions in Krasnoselskii's fixed point theorem (see [10, Lemma 2.2]) is similar to that in [10, Theorem 3.1] and [13, Theorem 3.1], hence it is omitted here. In terms of Krasnoselskii's fixed point theorem, there exists an $x \in \Omega_{1}$ such that $\left(U_{1}+V_{1}\right) x=x$, and then, for $t \in\left[T_{1}, \infty\right)_{\mathbb{T}}$, we have

$$
\begin{aligned}
x(t)= & \frac{3\left(1+p_{1} \eta\right) K R(t)}{4}-p(t) x(g(t)) \\
& +\int_{T_{1}}^{t} \int_{u_{n-1}}^{\infty} \int_{T_{1}}^{u_{n-2}} \int_{T_{1}}^{u_{n-3}} \cdots \int_{T_{1}}^{u_{1}} \frac{f\left(u_{0}, x\left(h\left(u_{0}\right)\right)\right)}{\prod_{i=1}^{n-1} r_{i}\left(u_{i}\right)} \Delta u_{0} \Delta u_{1} \cdots \Delta u_{n-1} .
\end{aligned}
$$


Since $x\left(h\left(u_{0}\right)\right) \leq K R\left(h\left(u_{0}\right)\right)$ for $u_{0} \in\left[T_{1}, \infty\right)_{\mathbb{T}}$ and

$$
\begin{gathered}
\lim _{t \rightarrow \infty} \frac{1}{R(t)} \int_{T_{1}}^{t} \int_{u_{n-1}}^{\infty} \int_{T_{1}}^{u_{n-2}} \int_{T_{1}}^{u_{n-3}} \cdots \int_{T_{1}}^{u_{1}} \frac{f\left(u_{0}, K R\left(h\left(u_{0}\right)\right)\right)}{\prod_{i=1}^{n-1} r_{i}\left(u_{i}\right)} \Delta u_{0} \Delta u_{1} \cdots \Delta u_{n-1} \\
\quad=\lim _{t \rightarrow \infty} \int_{t}^{\infty} \int_{T_{1}}^{u_{n-2}} \int_{T_{1}}^{u_{n-3}} \cdots \int_{T_{1}}^{u_{1}} \frac{f\left(u_{0}, K R\left(h\left(u_{0}\right)\right)\right)}{\prod_{i=1}^{n-2} r_{i}\left(u_{i}\right)} \Delta u_{0} \Delta u_{1} \cdots \Delta u_{n-2}=0,
\end{gathered}
$$

combining (C4) and Lemma 2.1, we get

$$
\lim _{t \rightarrow \infty} \frac{z(t)}{R(t)}=\frac{3\left(1+p_{1} \eta\right) K}{4}, \quad \lim _{t \rightarrow \infty} \frac{x(t)}{R(t)}=\frac{3\left(1+p_{1} \eta\right) K}{4\left(1+p_{0} \eta\right)}>0
$$

and $\lim _{t \rightarrow \infty} x(t)=\infty$.

Case 2. $-1<p_{0}<0$. We choose a $p_{1}$ and a $T_{0} \in\left[t_{0}, \infty\right)_{\mathbb{T}}$ such that $-p_{0}<p_{1}<\left(1-4 p_{0}\right) / 5<$ 1 and

$$
\frac{5 p_{1}-1}{4} \leq-p(t) \leq p_{1}<1, \quad t \in\left[T_{0}, \infty\right)_{\mathbb{T}}
$$

Moreover, define $U_{1}^{\prime}$ on $\Omega_{1}$ as follows:

$$
\left(U_{1}^{\prime} x\right)(t)= \begin{cases}-3 K p_{1} \eta R(t) / 4-p\left(T_{1}\right) x\left(g\left(T_{1}\right)\right) R(t) / R\left(T_{1}\right), & t \in\left[T_{0}, T_{1}\right)_{\mathbb{T}} \\ -3 K p_{1} \eta R(t) / 4-p(t) x(g(t)), & t \in\left[T_{1}, \infty\right)_{\mathbb{T}}\end{cases}
$$

Similarly to the proof of Case 1 , we deduce that

$$
\lim _{t \rightarrow \infty} \frac{z(t)}{R(t)}=\frac{3\left(1-p_{1} \eta\right) K}{4}, \quad \lim _{t \rightarrow \infty} \frac{x(t)}{R(t)}=\frac{3\left(1-p_{1} \eta\right) K}{4\left(1+p_{0} \eta\right)}>0
$$

and $\lim _{t \rightarrow \infty} x(t)=\infty$. The proof is complete.

In the following, the sufficient conditions for the types $A(b, 0)$ and $A(\infty, 0)$ are given in Theorems 3.2 and 3.3, respectively.

Theorem 3.2 If there exists a constant $K>0$ such that

$$
\int_{t_{0}}^{\infty} \int_{u_{n-1}}^{\infty} \int_{t_{0}}^{u_{n-2}} \int_{t_{0}}^{u_{n-3}} \cdots \int_{t_{0}}^{u_{1}} \frac{f\left(u_{0}, K\right)}{\prod_{i=1}^{n-1} r_{i}\left(u_{i}\right)} \Delta u_{0} \Delta u_{1} \cdots \Delta u_{n-1}<\infty,
$$

then (4) has an eventually positive solution $x \in A(b, 0)$, where $b$ is a positive constant.

Proof Suppose that there exists a constant $K>0$ such that (14) holds. Similarly as the proof of the sufficiency in Theorem 3.1, we consider two cases.

Case 1. $0 \leq p_{0}<1$. When $p_{0}>0$, taking $p_{1}$ chosen in Theorem 3.1 , then there exists a $T_{0} \in\left[t_{0}, \infty\right)_{\mathbb{T}}$ such that

$$
p(t)>0, \quad \frac{5 p_{1}-1}{4} \leq p(t) \leq p_{1}<1, \quad t \in\left[T_{0}, \infty\right)_{\mathbb{T}}
$$


and

$$
\int_{T_{0}}^{\infty} \int_{u_{n-1}}^{\infty} \int_{T_{0}}^{u_{n-2}} \int_{T_{0}}^{u_{n-3}} \cdots \int_{T_{0}}^{u_{1}} \frac{f\left(u_{0}, K\right)}{\prod_{i=1}^{n-1} r_{i}\left(u_{i}\right)} \Delta u_{0} \Delta u_{1} \cdots \Delta u_{n-1} \leq \frac{\left(1-p_{1}\right) K}{8} .
$$

When $p_{0}=0$, choose $p_{1}$ such that $|p(t)| \leq p_{1} \leq 1 / 13$ for $t \in\left[T_{0}, \infty\right)_{\mathbb{T}}$. There also exists a $T_{1} \in\left(T_{0}, \infty\right)_{\mathbb{T}}$ such that $g(t) \geq T_{0}$ and $h(t) \geq T_{0}$ for $t \in\left[T_{1}, \infty\right)_{\mathbb{T}}$.

Define a Banach space $\mathrm{BC}_{0}\left[T_{0}, \infty\right)_{\mathbb{T}}$ as $(5)$,

$$
\Omega_{2}=\left\{x \in \mathrm{BC}_{0}\left[T_{0}, \infty\right)_{\mathbb{T}}: \frac{K}{2} \leq x(t) \leq K\right\}
$$

and the operators $U_{2}$ and $V_{2}: \Omega_{2} \rightarrow \mathrm{BC}_{0}\left[T_{0}, \infty\right)_{\mathbb{T}}$ as follows:

$$
\begin{aligned}
& \left(U_{2} x\right)(t)= \begin{cases}\left(U_{2} x\right)\left(T_{1}\right), & t \in\left[T_{0}, T_{1}\right)_{\mathbb{T}}, \\
3 K p_{1} / 4-p(t) x(g(t)), & t \in\left[T_{1}, \infty\right)_{\mathbb{T}},\end{cases} \\
& \left(V_{2} x\right)(t)= \begin{cases}3 K / 4, \quad t \in\left[T_{0}, T_{1}\right)_{\mathbb{T}}, \\
3 K / 4+\int_{T_{1}}^{t} \int_{u_{n-1}}^{\infty} \int_{T_{1}}^{u_{n-2}} \int_{T_{1}}^{u_{n-3}} \cdots \int_{T_{1}}^{u_{1}} \frac{f\left(u_{0}, x\left(h\left(u_{0}\right)\right)\right)}{\prod_{i=1}^{n-1} r_{i}\left(u_{i}\right)} \Delta u_{0} \Delta u_{1} \cdots \Delta u_{n-1}, \\
t \in\left[T_{1}, \infty\right)_{\mathbb{T}} .\end{cases}
\end{aligned}
$$

Similarly, there exists an $x \in \Omega_{2}$ such that $\left(U_{2}+V_{2}\right) x=x$. For $t \in\left[T_{1}, \infty\right)_{\mathbb{T}}$, we have

$$
\begin{aligned}
x(t)= & \frac{3\left(1+p_{1}\right) K}{4}-p(t) x(g(t)) \\
& +\int_{T_{1}}^{t} \int_{u_{n-1}}^{\infty} \int_{T_{1}}^{u_{n-2}} \int_{T_{1}}^{u_{n-3}} \cdots \int_{T_{1}}^{u_{1}} \frac{f\left(u_{0}, x\left(h\left(u_{0}\right)\right)\right)}{\prod_{i=1}^{n-1} r_{i}\left(u_{i}\right)} \Delta u_{0} \Delta u_{1} \cdots \Delta u_{n-1} .
\end{aligned}
$$

Since

$$
\begin{aligned}
0 & <\int_{T_{1}}^{\infty} \int_{u_{n-1}}^{\infty} \int_{T_{1}}^{u_{n-2}} \int_{T_{1}}^{u_{n-3}} \cdots \int_{T_{1}}^{u_{1}} \frac{f\left(u_{0}, x\left(h\left(u_{0}\right)\right)\right)}{\prod_{i=1}^{n-1} r_{i}\left(u_{i}\right)} \Delta u_{0} \Delta u_{1} \cdots \Delta u_{n-1} \\
& \leq \int_{T_{1}}^{\infty} \int_{u_{n-1}}^{\infty} \int_{T_{1}}^{u_{n-2}} \int_{T_{1}}^{u_{n-3}} \cdots \int_{T_{1}}^{u_{1}} \frac{f\left(u_{0}, K\right)}{\prod_{i=1}^{n-1} r_{i}\left(u_{i}\right)} \Delta u_{0} \Delta u_{1} \cdots \Delta u_{n-1}<\infty,
\end{aligned}
$$

letting $t \rightarrow \infty$ in (15), we obtain $0<\lim _{t \rightarrow \infty} z(t)<\infty$. By Lemma 2.1, it follows that

$$
0<\lim _{t \rightarrow \infty} x(t)<\infty \text { and } \lim _{t \rightarrow \infty} \frac{x(t)}{R(t)}=0,
$$

which means that $x \in A(b, 0)$, where $b$ is a positive constant.

Case 2. $-1<p_{0}<0$. Define $U_{2}^{\prime}$ on $\Omega_{2}$ as

$$
\left(U_{2}^{\prime} x\right)(t)= \begin{cases}\left(U_{2}^{\prime} x\right)\left(T_{1}\right), & t \in\left[T_{0}, T_{1}\right)_{\mathbb{T}} \\ -3 K p_{1} / 4-p(t) x(g(t)), & t \in\left[T_{1}, \infty\right)_{\mathbb{T}}\end{cases}
$$

Similarly, we get a conclusion as in Case 1. The proof is complete. 
Theorem 3.3 If there exists a constant $M>0$ such that $|p(t) R(t)| \leq M$ for $t \in\left[t_{0}, \infty\right)_{\mathbb{T}}$,

$$
\begin{aligned}
& \int_{t_{0}}^{\infty} \int_{t_{0}}^{u_{n-2}} \int_{t_{0}}^{u_{n-3}} \cdots \int_{t_{0}}^{u_{1}} \frac{f\left(u_{0}, R\left(h\left(u_{0}\right)\right)\right)}{\prod_{i=1}^{n-2} r_{i}\left(u_{i}\right)} \Delta u_{0} \Delta u_{1} \cdots \Delta u_{n-2}<\infty, \\
& \int_{t_{0}}^{\infty} \int_{u_{n-1}}^{\infty} \int_{t_{0}}^{u_{n-2}} \int_{t_{0}}^{u_{n-3}} \cdots \int_{t_{0}}^{u_{1}} \frac{f\left(u_{0}, M+3 / 4\right)}{\prod_{i=1}^{n-1} r_{i}\left(u_{i}\right)} \Delta u_{0} \Delta u_{1} \cdots \Delta u_{n-1}=\infty,
\end{aligned}
$$

then (4) has an eventually positive solution $x \in A(\infty, 0)$.

Proof Suppose that there exists a constant $M>0$ such that $|p(t) R(t)| \leq M$ for $t \in\left[t_{0}, \infty\right)_{\mathbb{T}}$. Then we know $\lim _{t \rightarrow \infty} p(t)=p_{0}=0$. Choose a $T_{0} \in\left[t_{0}, \infty\right)_{\mathbb{T}}$ and a $p_{1}$ with $0<p_{1}<1$ satisfying

$$
|p(t)| \leq p_{1}<1, \quad 2 M+\frac{3}{2} \leq \frac{1}{4} R(t), \quad t \in\left[T_{0}, \infty\right)_{\mathbb{T}},
$$

and

$$
\int_{T_{0}}^{\infty} \int_{T_{0}}^{u_{n-2}} \int_{T_{0}}^{u_{n-3}} \cdots \int_{T_{0}}^{u_{1}} \frac{f\left(u_{0}, R\left(h\left(u_{0}\right)\right)\right)}{\prod_{i=1}^{n-2} r_{i}\left(u_{i}\right)} \Delta u_{0} \Delta u_{1} \cdots \Delta u_{n-2} \leq \frac{1-p_{1}}{8} .
$$

There exists a $T_{1} \in\left(T_{0}, \infty\right)_{\mathbb{T}}$ such that $g(t) \geq T_{0}$ and $h(t) \geq T_{0}$ for $t \in\left[T_{1}, \infty\right)_{\mathbb{T}}$.

Define a Banach space $\mathrm{BC}_{1}\left[T_{0}, \infty\right)_{\mathbb{T}}$ as (5),

$$
\Omega_{3}=\left\{x \in \mathrm{BC}_{1}\left[T_{0}, \infty\right)_{\mathbb{T}}: M+\frac{3}{4} \leq x(t) \leq R(t)\right\}
$$

and the operators $U_{3}$ and $V_{3}: \Omega_{3} \rightarrow \mathrm{BC}_{1}\left[T_{0}, \infty\right)_{\mathbb{T}}$ as follows:

$$
\begin{aligned}
& \left(U_{3} x\right)(t)= \begin{cases}M+3 / 4-p\left(T_{1}\right) x\left(g\left(T_{1}\right)\right) R(t) / R\left(T_{1}\right), & t \in\left[T_{0}, T_{1}\right)_{\mathbb{T}}, \\
M+3 / 4-p(t) x(g(t)), & t \in\left[T_{1}, \infty\right)_{\mathbb{T}},\end{cases} \\
& \left(V_{3} x\right)(t)=\left\{\begin{array}{cc}
M+3 / 4, & t \in\left[T_{0}, T_{1}\right)_{\mathbb{T}}, \\
M+3 / 4+\int_{T_{1}}^{t} \int_{u_{n-1}}^{\infty} \int_{T_{1}}^{u_{n-2}} \int_{T_{1}}^{u_{n-3}} \cdots \int_{T_{1}}^{u_{1}} \frac{f\left(u_{0}, x\left(h\left(u_{0}\right)\right)\right)}{\prod_{i=1}^{n-1} r_{i}\left(u_{i}\right)} \Delta u_{0} \Delta u_{1} \cdots \Delta u_{n-1}, \\
t \in\left[T_{1}, \infty\right)_{\mathbb{T}} .
\end{array}\right.
\end{aligned}
$$

Similarly, there exists an $x \in \Omega_{3}$ such that $\left(U_{3}+V_{3}\right) x=x$. For $t \in\left[T_{1}, \infty\right)_{\mathbb{T}}$, we have

$$
\begin{aligned}
x(t)= & 2 M+\frac{3}{2}-p(t) x(g(t)) \\
& +\int_{T_{1}}^{t} \int_{u_{n-1}}^{\infty} \int_{T_{1}}^{u_{n-2}} \int_{T_{1}}^{u_{n-3}} \cdots \int_{T_{1}}^{u_{1}} \frac{f\left(u_{0}, x\left(h\left(u_{0}\right)\right)\right)}{\prod_{i=1}^{n-1} r_{i}\left(u_{i}\right)} \Delta u_{0} \Delta u_{1} \cdots \Delta u_{n-1} .
\end{aligned}
$$

Since $M+3 / 4 \leq x\left(h\left(u_{0}\right)\right) \leq R\left(h\left(u_{0}\right)\right)$ for $u_{0} \in\left[T_{1}, \infty\right)_{\mathbb{T}}$, in view of (C3), (C4), (16), and (17), we obtain

$$
\lim _{t \rightarrow \infty} \int_{T_{1}}^{t} \int_{u_{n-1}}^{\infty} \int_{T_{1}}^{u_{n-2}} \int_{T_{1}}^{u_{n-3}} \cdots \int_{T_{1}}^{u_{1}} \frac{f\left(u_{0}, x\left(h\left(u_{0}\right)\right)\right)}{\prod_{i=1}^{n-1} r_{i}\left(u_{i}\right)} \Delta u_{0} \Delta u_{1} \cdots \Delta u_{n-1}=\infty
$$


and

$$
\begin{gathered}
\lim _{t \rightarrow \infty} \frac{1}{R(t)} \int_{T_{1}}^{t} \int_{u_{n-1}}^{\infty} \int_{T_{1}}^{u_{n-2}} \int_{T_{1}}^{u_{n-3}} \cdots \int_{T_{1}}^{u_{1}} \frac{f\left(u_{0}, R\left(h\left(u_{0}\right)\right)\right)}{\prod_{i=1}^{n-1} r_{i}\left(u_{i}\right)} \Delta u_{0} \Delta u_{1} \cdots \Delta u_{n-1} \\
\quad=\lim _{t \rightarrow \infty} \int_{t}^{\infty} \int_{T_{1}}^{u_{n-2}} \int_{T_{1}}^{u_{n-3}} \cdots \int_{T_{1}}^{u_{1}} \frac{f\left(u_{0}, R\left(h\left(u_{0}\right)\right)\right)}{\prod_{i=1}^{n-2} r_{i}\left(u_{i}\right)} \Delta u_{0} \Delta u_{1} \cdots \Delta u_{n-2}=0 .
\end{gathered}
$$

It is not difficult to see that $\lim _{t \rightarrow \infty} z(t)=\infty$ and $\lim _{t \rightarrow \infty} z(t) / R(t)=0$. Since $|p(t) x(g(t))| \leq$ $|p(t) R(t)| \leq M$ for $t \in\left[T_{1}, \infty\right)_{\mathbb{T}}$, we arrive at

$$
\lim _{t \rightarrow \infty} x(t)=\infty \quad \text { and } \quad \lim _{t \rightarrow \infty} \frac{x(t)}{R(t)}=0
$$

due to Lemma 2.1. The proof is complete.

Next, we present the necessary conditions for the types $A(b, 0)$ and $A(\infty, 0)$. An additional assumption

$$
\int_{t_{0}}^{\infty} \int_{u_{n-1}}^{\infty} \int_{t_{0}}^{u_{n-2}} \int_{t_{0}}^{u_{n-3}} \cdots \int_{t_{0}}^{u_{n-k+1}} \frac{\Delta u_{n-k} \Delta u_{n-k+1} \cdots \Delta u_{n-1}}{\prod_{i=n-k}^{n-1} r_{i}\left(u_{i}\right)}<\infty
$$

is required, where $2 \leq k \leq n-1$. Then we deduce Theorems 3.4 and 3.5.

Theorem 3.4 Suppose that (18) holds for all $2 \leq k \leq n-1$. If (4) has an eventually positive solution $x \in A(b, 0)$, where $b$ is a positive constant, then there exists a constant $K>0$ such that (14) holds.

Proof Suppose that $x \in A(b, 0)$ is an eventually positive solution to (4), where $b$ is a positive constant. Then we have

$$
\lim _{t \rightarrow \infty} z(t)=\left(1+p_{0}\right) b, \quad \lim _{t \rightarrow \infty} r_{n-1}(t) z^{\Delta}(t)=\lim _{t \rightarrow \infty} \frac{z(t)}{R(t)}=0,
$$

and there exists a $t_{1} \in\left[t_{0}, \infty\right)_{\mathbb{T}}$ such that $x(t) \geq b / 2, x(g(t)) \geq b / 2$, and $x(h(t)) \geq b / 2, t \in$ $\left[t_{1}, \infty\right)_{\mathbb{T}}$. As by the proof in Theorem 3.1, for all $n \geq 3$, there exists a $t_{2} \in\left[t_{1}, \infty\right)_{\mathbb{T}}$ such that

$$
\begin{aligned}
& r_{n-1}\left(u_{n-1}\right) z^{\Delta}\left(u_{n-1}\right)-r_{n-1}\left(t_{2}\right) z^{\Delta}\left(t_{2}\right) \\
& =\sum_{k=2}^{n-1} R_{k}\left(t_{1}, x\left(t_{1}\right)\right) \int_{t_{2}}^{u_{n-1}} \int_{t_{1}}^{u_{n-2}} \int_{t_{1}}^{u_{n-3}} \cdots \int_{t_{1}}^{u_{n-k+1}} \frac{\Delta u_{n-k} \Delta u_{n-k+1} \cdots \Delta u_{n-2}}{\prod_{i=n-k}^{n-2} r_{i}\left(u_{i}\right)} \\
& \quad-\int_{t_{2}}^{u_{n-1}} \int_{t_{1}}^{u_{n-2}} \int_{t_{1}}^{u_{n-3}} \cdots \int_{t_{1}}^{u_{1}} \frac{f\left(u_{0}, x\left(h\left(u_{0}\right)\right)\right)}{\prod_{i=1}^{n-2} r_{i}\left(u_{i}\right)} \Delta u_{0} \Delta u_{1} \cdots \Delta u_{n-2} .
\end{aligned}
$$

Letting $u_{n-1} \rightarrow \infty$, we obtain

$$
\begin{aligned}
& -r_{n-1}\left(t_{2}\right) z^{\Delta}\left(t_{2}\right) \\
& =\sum_{k=2}^{n-1} R_{k}\left(t_{1}, x\left(t_{1}\right)\right) \int_{t_{2}}^{\infty} \int_{t_{1}}^{u_{n-2}} \int_{t_{1}}^{u_{n-3}} \cdots \int_{t_{1}}^{u_{n-k+1}} \frac{\Delta u_{n-k} \Delta u_{n-k+1} \cdots \Delta u_{n-2}}{\prod_{i=n-k}^{n-2} r_{i}\left(u_{i}\right)} \\
& \quad-\int_{t_{2}}^{\infty} \int_{t_{1}}^{u_{n-2}} \int_{t_{1}}^{u_{n-3}} \cdots \int_{t_{1}}^{u_{1}} \frac{f\left(u_{0}, x\left(h\left(u_{0}\right)\right)\right)}{\prod_{i=1}^{n-2} r_{i}\left(u_{i}\right)} \Delta u_{0} \Delta u_{1} \cdots \Delta u_{n-2} .
\end{aligned}
$$


From (18), it follows that

$$
\int_{t_{2}}^{\infty} \int_{t_{1}}^{u_{n-2}} \int_{t_{1}}^{u_{n-3}} \cdots \int_{t_{1}}^{u_{1}} \frac{f\left(u_{0}, x\left(h\left(u_{0}\right)\right)\right)}{\prod_{i=1}^{n-2} r_{i}\left(u_{i}\right)} \Delta u_{0} \Delta u_{1} \cdots \Delta u_{n-2}<\infty .
$$

Since $x\left(h\left(u_{0}\right)\right) \geq b / 2, u_{0} \in\left[t_{1}, \infty\right)_{\mathbb{T}}$, by $(\mathrm{C} 4)$ we get

$$
\int_{t_{2}}^{\infty} \int_{t_{1}}^{u_{n-2}} \int_{t_{1}}^{u_{n-3}} \cdots \int_{t_{1}}^{u_{1}} \frac{f\left(u_{0}, b / 2\right)}{\prod_{i=1}^{n-2} r_{i}\left(u_{i}\right)} \Delta u_{0} \Delta u_{1} \cdots \Delta u_{n-2}<\infty .
$$

Substituting $u_{n-1}$ for $t_{2}$ into (19), we have

$$
\begin{aligned}
& z^{\Delta}\left(u_{n-1}\right) \\
& =-\sum_{k=2}^{n-1} R_{k}\left(t_{1}, x\left(t_{1}\right)\right) \int_{u_{n-1}}^{\infty} \int_{t_{1}}^{u_{n-2}} \int_{t_{1}}^{u_{n-3}} \cdots \int_{t_{1}}^{u_{n-k+1}} \frac{\Delta u_{n-k} \Delta u_{n-k+1} \cdots \Delta u_{n-2}}{\prod_{i=n-k}^{n-1} r_{i}\left(u_{i}\right)} \\
& \quad+\int_{u_{n-1}}^{\infty} \int_{t_{1}}^{u_{n-2}} \int_{t_{1}}^{u_{n-3}} \cdots \int_{t_{1}}^{u_{1}} \frac{f\left(u_{0}, x\left(h\left(u_{0}\right)\right)\right)}{\prod_{i=1}^{n-1} r_{i}\left(u_{i}\right)} \Delta u_{0} \Delta u_{1} \cdots \Delta u_{n-2} .
\end{aligned}
$$

Integrating (22) from $t_{2}$ to $t, t \in\left[\sigma\left(t_{2}\right), \infty\right)_{\mathbb{T}}$, we obtain

$$
\begin{aligned}
z(t)- & z\left(t_{2}\right) \\
=- & \sum_{k=2}^{n-1} R_{k}\left(t_{1}, x\left(t_{1}\right)\right) \int_{t_{2}}^{t} \int_{u_{n-1}}^{\infty} \int_{t_{1}}^{u_{n-2}} \int_{t_{1}}^{u_{n-3}} \cdots \int_{t_{1}}^{u_{n-k+1}} \frac{\Delta u_{n-k} \Delta u_{n-k+1} \cdots \Delta u_{n-1}}{\prod_{i=n-k}^{n-1} r_{i}\left(u_{i}\right)} \\
& +\int_{t_{2}}^{t} \int_{u_{n-1}}^{\infty} \int_{t_{1}}^{u_{n-2}} \int_{t_{1}}^{u_{n-3}} \cdots \int_{t_{1}}^{u_{1}} \frac{f\left(u_{0}, x\left(h\left(u_{0}\right)\right)\right)}{\prod_{i=1}^{n-1} r_{i}\left(u_{i}\right)} \Delta u_{0} \Delta u_{1} \cdots \Delta u_{n-1} .
\end{aligned}
$$

Letting $t \rightarrow \infty$, by (18) we deduce that

$$
\int_{t_{2}}^{\infty} \int_{u_{n-1}}^{\infty} \int_{t_{1}}^{u_{n-2}} \int_{t_{1}}^{u_{n-3}} \cdots \int_{t_{1}}^{u_{1}} \frac{f\left(u_{0}, b / 2\right)}{\prod_{i=1}^{n-1} r_{i}\left(u_{i}\right)} \Delta u_{0} \Delta u_{1} \cdots \Delta u_{n-1}<\infty,
$$

which covers (21) and implies that (14) holds. The proof is complete.

Theorem 3.5 Suppose that (18) holds for all $2 \leq k \leq n-1$. If (4) has an eventually positive solution $x \in A(\infty, 0)$, then

$$
\int_{t_{0}}^{\infty} \int_{t_{0}}^{u_{n-2}} \int_{t_{0}}^{u_{n-3}} \cdots \int_{t_{0}}^{u_{1}} \frac{f\left(u_{0}, 3 / 4\right)}{\prod_{i=1}^{n-2} r_{i}\left(u_{i}\right)} \Delta u_{0} \Delta u_{1} \cdots \Delta u_{n-2}<\infty
$$

and

$$
\int_{t_{0}}^{\infty} \int_{u_{n-1}}^{\infty} \int_{t_{0}}^{u_{n-2}} \int_{t_{0}}^{u_{n-3}} \cdots \int_{t_{0}}^{u_{1}} \frac{f\left(u_{0}, R\left(h\left(u_{0}\right)\right)\right)}{\prod_{i=1}^{n-1} r_{i}\left(u_{i}\right)} \Delta u_{0} \Delta u_{1} \cdots \Delta u_{n-1}=\infty
$$

Proof Suppose that $x \in A(\infty, 0)$ is an eventually positive solution to (4). Then we have

$$
\lim _{t \rightarrow \infty} z(t)=\infty, \quad \lim _{t \rightarrow \infty} r_{n-1}(t) z^{\Delta}(t)=\lim _{t \rightarrow \infty} \frac{z(t)}{R(t)}=0,
$$


and there exists a $T \in\left[t_{0}, \infty\right)_{\mathbb{T}}$ satisfying that $3 / 4 \leq x(t) \leq R(t)$ for $t \in[T, \infty)_{\mathbb{T}}$. From $(\mathrm{C} 3)$, there exists a $t_{1} \in[T, \infty)_{\mathbb{T}}$ such that $g(t) \geq T$ and $h(t) \geq T, t \in\left[t_{1}, \infty\right)_{\mathbb{T}}$. As the proof in Theorem 3.4, there exists a $t_{2} \in\left[t_{1}, \infty\right)_{\mathbb{T}}$ such that (20) holds. In view of (C4), we get

$$
\int_{t_{2}}^{\infty} \int_{t_{1}}^{u_{n-2}} \int_{t_{1}}^{u_{n-3}} \cdots \int_{t_{1}}^{u_{1}} \frac{f\left(u_{0}, 3 / 4\right)}{\prod_{i=1}^{n-2} r_{i}\left(u_{i}\right)} \Delta u_{0} \Delta u_{1} \cdots \Delta u_{n-2}<\infty
$$

that is, (24) holds. Proceeding as in the proof of Theorem 3.4, we arrive at (23). Letting $t \rightarrow \infty$, by (C4) and (18) we obtain

$$
\int_{t_{2}}^{\infty} \int_{u_{n-1}}^{\infty} \int_{t_{1}}^{u_{n-2}} \int_{t_{1}}^{u_{n-3}} \cdots \int_{t_{1}}^{u_{1}} \frac{f\left(u_{0}, R\left(h\left(u_{0}\right)\right)\right)}{\prod_{i=1}^{n-1} r_{i}\left(u_{i}\right)} \Delta u_{0} \Delta u_{1} \cdots \Delta u_{n-1}=\infty,
$$

which implies that (25) holds. The proof is complete.

Remark 3.6 From Theorems 3.2 and 3.4, it is clear to see that, when (18) holds for all $2 \leq k \leq n-1,(4)$ has an eventually positive solution $x \in A(b, 0)$ if and only if there exists a constant $K>0$ such that (14) holds, where $b$ is a positive constant.

Remark 3.7 Assume that $x \in A(0,0)$ is an eventually positive solution to (4). The asymptotic behavior of $x$ is more complex than that in other types. Hence, it is not easy to find a sufficient and necessary condition for the type $A(0,0)$. Some sufficient conditions were presented to ensure that (4) has an eventually positive solution $x \in A(0,0)$, we refer the reader to [13, Theorems 3.2 and 3.3].

Remark 3.8 It is obvious that the theorems reported in this paper cover those in [12] when $n=3$. Furthermore, they are consistent with the conclusions in [5] when $n=2$. Besides, they also complement and improve the results in [13].

\section{Examples}

In this section, we present two typical examples to illustrate the applications of our results.

Example 4.1 Let $\mathbb{T}=\bigcup_{n=0}^{\infty}[4 n, 4 n+3]$. For $t \in[4, \infty)_{\mathbb{T}}$, consider the $n$ th-order dynamic equation

$$
R_{n}(t, x(t))+\frac{x(t)}{t^{2}}=0
$$

where $n \geq 4$,

$$
R_{k}(t, x(t))= \begin{cases}x(t)+(t-1) x(t-4) /(3 t), & k=0, \\ t^{\alpha_{n-k}} R_{k-1}^{\Delta}(t, x(t)), & 1 \leq k \leq n-1, \\ R_{n-1}^{\Delta}(t, x(t)), & k=n,\end{cases}
$$

$\alpha_{1}>2, \alpha_{n-2}>3$, and $\alpha_{n-1}=-1$. If $n \geq 5$, then let $\alpha_{i}>1, i=2,3, \ldots, n-3$.

Here, $r_{i}(t)=t^{\alpha_{i}}, i=1,2, \ldots, n-1, p(t)=(t-1) /(3 t), g(t)=t-4, h(t)=t$, and $f(t, x)=x / t^{2}$. It is not difficult to see that $(\mathrm{C} 1)-(\mathrm{C} 4)$ are all satisfied. Since

$$
R(t)=1+\int_{4}^{t} s \Delta s=O\left(t^{2}\right),
$$


it follows that

$$
\begin{aligned}
& \int_{4}^{\infty} \int_{4}^{u_{n-2}} \int_{4}^{u_{n-3}} \cdots \int_{4}^{u_{1}} \frac{f\left(u_{0}, R\left(h\left(u_{0}\right)\right)\right)}{\prod_{i=1}^{n-2} r_{i}\left(u_{i}\right)} \Delta u_{0} \Delta u_{1} \cdots \Delta u_{n-2} \\
& <O(1) \cdot \int_{4}^{\infty} \int_{4}^{\infty} \cdots \int_{4}^{\infty} \frac{\Delta u_{1} \Delta u_{2} \cdots \Delta u_{n-2}}{u_{1}^{\alpha_{1}-1} \cdot \prod_{i=2}^{n-2} u_{i}^{\alpha_{i}}} \\
& =O(1) \cdot \int_{4}^{\infty} \frac{\Delta u_{1}}{u_{1}^{\alpha_{1}-1}} \cdot \prod_{i=2}^{n-2} \int_{4}^{\infty} \frac{\Delta u_{i}}{u_{i}^{\alpha_{i}}<\infty,} \\
& \int_{4}^{\infty} \int_{u_{n-1}}^{\infty} \int_{4}^{u_{n-2}} \int_{4}^{u_{n-3}} \cdots \int_{4}^{u_{1}} \frac{f\left(u_{0}, 1\right)}{\prod_{i=1}^{n-1} r_{i}\left(u_{i}\right)} \Delta u_{0} \Delta u_{1} \cdots \Delta u_{n-1} \\
& =\int_{4}^{\infty} \int_{u_{n-1}}^{\infty} \int_{4}^{u_{n-2}} \int_{4}^{u_{n-3}} \cdots \int_{4}^{u_{1}} \frac{\Delta u_{0} \Delta u_{1} \cdots \Delta u_{n-1}}{u_{0}^{2} \cdot \prod_{i=1}^{n-1} r_{i}\left(u_{i}\right)} \\
& <O(1) \cdot \int_{4}^{\infty} \int_{u_{n-1}}^{\infty} \frac{u_{n-1}}{u_{n-2}^{\alpha_{n-2}}} \Delta u_{n-2} \Delta u_{n-1} \cdot \prod_{i=1}^{n-3} \int_{4}^{\infty} \frac{\Delta u_{i}}{u_{i}^{\alpha_{i}}} \\
& =O(1) \cdot \int_{4}^{\infty} \frac{\Delta u_{n-1}}{u_{n-1}^{\alpha_{n-2}-2}} \cdot \prod_{i=1}^{n-3} \int_{4}^{\infty} \frac{\Delta u_{i}}{u_{i}^{\alpha_{i}}<\infty,}
\end{aligned}
$$

and

$$
\begin{aligned}
& \int_{4}^{\infty} \int_{u_{n-1}}^{\infty} \int_{4}^{u_{n-2}} \int_{4}^{u_{n-3}} \cdots \int_{4}^{u_{1}} \frac{f\left(u_{0}, R\left(h\left(u_{0}\right)\right)\right)}{\prod_{i=1}^{n-1} r_{i}\left(u_{i}\right)} \Delta u_{0} \Delta u_{1} \cdots \Delta u_{n-1} \\
& =O(1) \cdot \int_{4}^{\infty} \int_{u_{n-1}}^{\infty} \int_{4}^{u_{n-2}} \int_{4}^{u_{n-3}} \cdots \int_{4}^{u_{1}} \frac{\Delta u_{0} \Delta u_{1} \cdots \Delta u_{n-1}}{\prod_{i=1}^{n-1} r_{i}\left(u_{i}\right)} \\
& <O(1) \cdot \int_{4}^{\infty} \int_{u_{n-1}}^{\infty} \frac{u_{n-1}}{u_{n-2}^{\alpha_{n-2}}} \Delta u_{n-2} \Delta u_{n-1} \cdot \int_{4}^{\infty} \frac{\Delta u_{1}}{u_{1}^{\alpha_{1}-1}} \cdot \prod_{i=2}^{n-3} \int_{4}^{\infty} \frac{\Delta u_{i}}{u_{i}^{\alpha_{i}}}<\infty,
\end{aligned}
$$

where $\prod_{i=2}^{n-3} \int_{4}^{\infty} 1 / u_{i}^{\alpha_{i}} \Delta u_{i}$ is deleted when $n=4$. The results mean that (11) and (14) hold, but (25) is not satisfied. Therefore, by Theorems 3.1 and 3.2, we conclude that (26) has eventually positive solutions $x_{1} \in A\left(\infty, b_{1}\right)$ and $x_{2} \in A\left(b_{2}, 0\right)$, where $b_{1}$ and $b_{2}$ are both positive constants. However, (26) has no eventually positive solutions in $A(\infty, 0)$ due to Theorem 3.5. In addition, for $n=3$, letting $r_{1}(t)=t^{\alpha_{1}}$ and $r_{2}(t)=1 / t$, where $\alpha_{1}>4$, we can get similar conclusions.

Example 4.2 Let $\mathbb{T}=[1, \infty)_{\mathbb{R}}$. For $t \in[3, \infty)_{\mathbb{T}}$, consider the fourth-order dynamic equation

$$
R_{4}(t, x(t))+\frac{x(\sqrt{t})}{t^{\beta}}=0,
$$

where

$$
R_{k}(t, x(t))= \begin{cases}x(t)-x(t / 3) / t, & k=0, \\ t^{\alpha_{4-k}} R_{k-1}^{\Delta}(t, x(t)), & 1 \leq k \leq 3, \\ R_{3}^{\Delta}(t, x(t)), & k=4,\end{cases}
$$

$\alpha_{1}=\alpha_{2}=2, \alpha_{3}=0$, and $\beta \geq 3 / 2$. 
Here, $r_{1}(t)=r_{2}(t)=t^{2}, r_{3}(t)=1, p(t)=-1 / t, g(t)=t / 3, h(t)=\sqrt{t}$, and $f(t, x)=x / t^{\beta}$. Obviously, conditions $(\mathrm{C} 1)-(\mathrm{C} 4)$ are satisfied. Since

$$
R(t)=1+\int_{3}^{t} d s=t-2,
$$

taking $M=5 / 4$, we obtain

$$
\begin{aligned}
& |p(t) R(t)|=\frac{t-2}{t}<M, \\
& \int_{3}^{\infty} \int_{3}^{u_{2}} \int_{3}^{u_{1}} \frac{f\left(u_{0}, R\left(h\left(u_{0}\right)\right)\right)}{u_{1}^{2} u_{2}^{2}} d u_{0} d u_{1} d u_{2}<\int_{3}^{\infty} \int_{3}^{u_{2}} \int_{3}^{u_{1}} \frac{d u_{0} d u_{1} d u_{2}}{u_{0}^{\beta-1 / 2} u_{1}^{2} u_{2}^{2}}<\infty
\end{aligned}
$$

and

$$
\begin{aligned}
& \int_{3}^{\infty} \int_{u_{3}}^{\infty} \int_{3}^{u_{2}} \int_{3}^{u_{1}} \frac{f\left(u_{0}, M+3 / 4\right)}{u_{1}^{2} u_{2}^{2}} d u_{0} d u_{1} d u_{2} d u_{3} \\
& \quad=\int_{3}^{\infty} \int_{u_{3}}^{\infty} \int_{3}^{u_{2}} \int_{3}^{u_{1}} \frac{2}{u_{0}^{\beta} u_{1}^{2} u_{2}^{2}} d u_{0} d u_{1} d u_{2} d u_{3}=\infty
\end{aligned}
$$

Therefore, we deduce that (27) has an eventually positive solution $x \in A(\infty, 0)$ in terms of Theorem 3.3.

\section{Acknowledgements}

The authors express their sincere gratitude to the editors and two anonymous referees for the careful reading of the original manuscript and useful comments, which helped to improve the presentation of the results and accentuate important details.

\section{Funding}

The research of the first author was supported by the National Natural Science Foundation of P.R. China (Grant No. 11671406) and Natural Science Program for Young Creative Talents of Innovation Enhancing College Project of Department of Education of Guangdong Province (Grant Nos. 2017GKQNCX111 and 2018-KJZX039). The research of the second author was supported by PGI 03-2020 DIUMCE. The research of the third author was supported by the Slovak Research and Development Agency (Grant No. APV-18-0373). The research of the fourth author was supported by the National Natural Science Foundation of P.R. China (Grant No. 61503171), China Postdoctoral Science Foundation (Grant No. 2015M582091), and Natural Science Foundation of Shandong Province (Grant No. ZR2016JL021).

\section{Availability of data and materials}

Data sharing not applicable to this article as no datasets were generated or analysed during the current study.

\section{Competing interests}

The authors declare that they have no competing interests.

\section{Authors' contributions}

All four authors contributed equally to this work. They all read and approved the final version of the manuscript.

\section{Author details}

'School of Humanities, Shunde Polytechnic, Foshan, P.R. China. ${ }^{2}$ Departamento de Matemática, Facultad de Ciencias Básicas, Universidad Metropolitana de Ciencias de la Educación, Santiago, Chile. ${ }^{3}$ Department of Mathematics and Theoretical Informatics, Faculty of Electrical Engineering and Informatics, Technical University of Košice, Košice, Slovakia.

${ }^{4}$ School of Control Science and Engineering, Shandong University, Jinan, P.R. China.

\section{Publisher's Note}

Springer Nature remains neutral with regard to jurisdictional claims in published maps and institutional affiliations. 


\section{References}

1. Agarwal, R.P., Bohner, M.: Basic calculus on time scales and some of its applications. Results Math. 35, 3-22 (1999)

2. Agarwal, R.P., Bohner, M., O'Regan, D., Peterson, A.: Dynamic equations on time scales: a survey. J. Comput. Appl. Math. 141, 1-26 (2002)

3. Bohner, M., Peterson, A.: Dynamic Equations on Time Scales: An Introduction with Applications. Birkhäuser, Boston (2001)

4. Bohner, M., Peterson, A.: Advances in Dynamic Equations on Time Scales. Birkhäuser, Boston (2003)

5. Deng, X.-H., Wang, Q.-R.: Nonoscillatory solutions to second-order neutral functional dynamic equations on time scales. Commun. Appl. Anal. 18, 261-280 (2014)

6. Gao, J., Wang, Q.R.: Existence of nonoscillatory solutions to second-order nonlinear neutral dynamic equations on time scales. Rocky Mt. J. Math. 43, 1521-1535 (2013)

7. Hilger, S.: Ein Maßkettenkalkül mit Anwendung auf Zentrumsmannigfaltigkeiten. Ph.D. thesis, Universität Würzburg, Würzburg, Germany (1988)

8. Hilger, S.: Analysis on measure chains - a unified approach to continuous and discrete calculus. Results Math. 18, $18-56(1990)$

9. Li, T.X., Han, Z.L., Sun, S.R., Yang, D.W.: Existence of nonoscillatory solutions to second-order neutral delay dynamic equations on time scales. Adv. Differ. Equ. 2009, Article ID 562329 (2009)

10. Qiu, Y.-C.: Nonoscillatory solutions to third-order neutral dynamic equations on time scales. Adv. Differ. Equ. 2014, 309 (2014)

11. Qiu, Y.-C., Jadlovská, I., Lassoued, D., Li, T.X.: Nonoscillatory solutions to higher-order nonlinear neutral dynamic equations. Symmetry 11, 302 (2019)

12. Qiu, Y.-C., Wang, H.X., Jiang, C.M., Li, T.X.: Existence of nonoscillatory solutions to third-order neutral functional dynamic equations on time scales. J. Nonlinear Sci. Appl. 11, 274-287 (2018)

13. Qiu, Y.-C., Wang, Q.-R.: Existence of nonoscillatory solutions to higher-order nonlinear neutral dynamic equations on time scales. Bull. Malays. Math. Sci. Soc. 41, 1935-1952 (2018)

14. Qiu, Y.-C., Zada, A., Tang, S.H., Li, T.X.: Existence of nonoscillatory solutions to nonlinear third-order neutral dynamic equations on time scales. J. Nonlinear Sci. Appl. 10, 4352-4363 (2017)

15. Zhu, Z.-Q., Wang, Q.-R.: Existence of nonoscillatory solutions to neutral dynamic equations on time scales. J. Math. Anal. Appl. 335, 751-762 (2007)

\section{Submit your manuscript to a SpringerOpen ${ }^{\circ}$ journal and benefit from:}

- Convenient online submission

- Rigorous peer review

- Open access: articles freely available online

- High visibility within the field

- Retaining the copyright to your article

Submit your next manuscript at $\boldsymbol{~ s p r i n g e r o p e n . c o m ~}$ 